\title{
LINEARITY AND WEAK CONVERGENCE ON THE BOUNDARY OF NUMERICAL RANGE
}

\author{
K. C. DAS and B. D. CRAVEN \\ (Received 7 August 1981; revised 29 September 1982) \\ Communicated by E. Strzelecki
}

\begin{abstract}
Stampfli and Embry have shown that a point of the numerical range of an operator is extreme if and only if a set of vectors corresponding to it is linear. This is generalized here to show that a point of the closure of the numerical range is extreme if and only if a corresponding set of sequences forms a linear space. A more geometric alternative proof is given for a theorem of Das and Garske concerning weak convergence to zero at the unattained extreme points of the closure of the numerical range. The result is shown to hold also for lone extreme points of the numerical range which lie on line segments on its boundary. Further, a bound is obtained on the norm of the weak limit of the weakly convergent sequences corresponding to points on a line segment on the boundary of numerical range.
\end{abstract}

1980 Mathematics subject classification (Amer. Math. Soc.): 47 A 12.

\section{Introduction}

Let $H$ be a complex separable Hilbert space with inner product $(\cdot, \cdot)$ and norm \| \|. For $T \in B(H)$, the algebra of bounded linear operators, the numerical range, its closure, and its topological boundary are denoted, in order, by $W(T), W(T)^{-}$ and $\partial W(T)$. For any complex number $z$, define $M_{z}(T)=\{f: f \in H$ and $(T f, f)$ $-z(f, f)=0\}$. Stampfli [1966] has shown that $M_{z}(T)$ is linear if $z$ is an extreme point of $W(T)$. An alternative proof of this result occurs in Embry [1975]. Embry [1970] established the converse of this result, namely, if $M_{z}(T)$ is linear then $z$ must be an extreme point of $W(T)$. Since the numerical range $W(T)$ is only a convex set in the plane, not necessarily closed, the results of Stampfli and Embry are inapplicable to the unattained boundary points of the numerical range.

(C) 1983 Australian Mathematical Society $0263-6115 / 83 \$ A 2.00+0.00$ 
Therefore, a generalization of their results that may hold for all extreme points of the closure of the numerical range seems to be called for. Das [1977] has shown that if $\mu$ is an unattained bare point on the boundary of $W(T)^{-}$, then all weakly convergent sequences of unit vectors for which $\left(T f_{n}, f_{n}\right) \rightarrow \mu$, must have zero only as their weak limit. Garske [1979] has generalized it to all unattained extreme boundary points of $W(T)^{-}$. He has also given an example to show that this property does not hold in general for all boundary points of $W(T)^{-}$. We have, however, shown here that the above result holds also for lone extreme points of $W(T)$ lying on line segments on $\partial W(T)$. The example of Garske shows that if $\left(T f_{n}, f_{n}\right) \rightarrow \mu, f_{n} \rightarrow f,\left\|f_{n}\right\|=1$, where $\mu$ is a point on a line segment of $W(T)$, then it is possible that $(T f, f) /(f, f)=\lambda \neq \mu$. We show here that in such a case $\lambda$ must be a point on the same line segment and the norm of the weak limit must have an upper bound as given in Theorem 4.

\section{Linearity on $\partial W(T)$}

For any complex number $z$, define

$$
N_{z}(T)=\left\{\left\{f_{n}\right\} \in l^{\infty}(H):\left(T f_{n}, f_{n}\right)-z\left(f_{n}, f_{n}\right) \rightarrow 0\right\} .
$$

If we look upon $H$ as embedded in $l^{\infty}(H)$ with the correspondence $f \rightarrow(f, f, \ldots)$, then $M_{z}(T)$ embedded in an evidently similar manner will be a subset of $N_{z}(T)$ if $z \in W(T)$. For unattained boundary points of $W(T), M_{z}(T)$ will consist of the zero vector while $N_{z}(T)$ will be nontrivial set of sequences. It will now be our endeavour to prove the following theorem:

THEOREM 1. $N_{z}(T)$ is a subspace of $l^{\infty}(H)$ if and only if $z$ is an extreme point of $W(T)^{-}$.

Proof. First we prove that $N_{z}(T)$ is a subspace if $z$ is an extreme point of $W(T)^{-}$. To that end we carry out the standard reduction by defining $A=$ $\exp (i \theta)(T-z I)$, where $\theta$ is so chosen that $W(A)$ lies on the right half-plane. Note that $\operatorname{Re} A \geqslant 0$. Evidently, $N_{z}(T)$ is linear and homogeneous if and only if $N_{0}(A)$ is so.

Homogeneity being clear, we need prove only linearity. Let $\left\{f_{n}\right\},\left\{g_{n}\right\} \in N_{0}(A)$. So $\left(A f_{n}+A^{*} f_{n}, f_{n}\right) \rightarrow 0$ and hence $h_{n}=A f_{n}+A^{*} f_{n} \rightarrow 0$, by virtue of positivity of $\operatorname{Re} A$. Now $\left(A f_{n}+A g_{n}, f_{n}+g_{n}\right)=k_{n}+2 i \operatorname{Im}\left(A f_{n}, g\right)$, where $k_{n}=\left(A f_{n}, f_{n}\right)+$ $\left(A g_{n}, g_{n}\right)+\left(g_{n}, h_{n}\right)$. Clearly $k_{n} \rightarrow 0$. If $\operatorname{Im}\left(A f_{n}, g_{n}\right)$ does not also tend to zero, we get a contradiction as shown below.

Case I. $\left\|f_{n}+g_{n}\right\| \geqslant K>0$ and $\left\|f_{n}-g_{n}\right\| \geqslant K^{\prime}>0$ for all $n$. By our hypothesis, $\operatorname{Im}\left(A f_{n}, g_{n}\right) /\left\|f_{n}+g_{n}\right\|^{2}$ is bounded and we can, therefore, always choose a 
subsequence such that $\operatorname{Im}\left(A f_{n^{\prime}}, g_{n^{\prime}}\right) /\left\|f_{n^{\prime}}+g_{n^{\prime}}\right\|^{2}$ converges to a nonzero number $a$. Assume that such a choice has already been made for simplicity of notations. Therefore $\left(A f_{n}+A g_{n}, f_{n}+g_{n}\right) /\left(f_{n}+g_{n}, f_{n}+g_{n}\right) \rightarrow 2 i a$. Let us say, for the sake of definiteness, that $a$ is positive. Consider again $\left(A f_{n}-A g_{n}, f_{n}-g_{n}\right) /\left(f_{n}-\right.$ $\left.g_{n}, f_{n}-g_{n}\right)=\left(t_{n}-2 i \operatorname{Im}\left(A f_{n}, g_{n}\right)\right) /\left(f_{n}-g_{n}, f_{n}-g_{n}\right)$, where $t_{n}=\left(A f_{n}, f_{n}\right)+$ $\left(A g_{n}, g_{n}\right)-\left(g_{n}, h_{n}\right)$. Clearly $t_{n} \rightarrow 0$. Since $\operatorname{Im}\left(A f_{n}, g_{n}\right) /\left\|f_{n}-g_{n}\right\|^{2}=$ $\left(\operatorname{Im}\left(A f_{n}, g_{n}\right) /\left\|f_{n}+g_{n}\right\|^{2}\right) \cdot\left(\left\|f_{n}+g_{n}\right\|^{2} /\left\|f_{n}-g_{n}\right\|^{2}\right)$, we see that it is always possible to extract a subsequence such that $2 i \operatorname{Im}\left(A f_{n^{\prime}}, g_{n^{\prime}}\right) /\left\|f_{n^{\prime}}-g_{n^{\prime}}\right\| \rightarrow 2 i b$, where $b>0$. Hence $W(T)^{-}$contains $2 i a$ and $-2 i b$ on the boundary contradicting that zero is an extreme point of $W(T)^{-}$.

Case II. $\left\|f_{n}+g_{n}\right\|\left\|f_{n}-g_{n}\right\|$ is not bounded away from zero. Consider the disjoint partition of the set $\{n\}$ of all natural numbers such that $\{n\}=\{n\} \cup\left\{u^{\prime \prime}\right\}$ and $\min \left\{\left\|f_{n^{\prime}}+g_{n^{\prime}}\right\|,\left\|f_{n^{\prime}}-g_{n^{\prime}}\right\|\right\}<\varepsilon / 2 M\|A\|$, where $\left\|f_{n}\right\| \leqslant M$ for all $n$, with $\left\|f_{n^{\prime \prime}}+g_{n^{\prime \prime}}\right\|,\left\|f_{n^{\prime \prime}}-g_{n^{\prime \prime}}\right\|$ bounded away from zero. Since $\left|\left(A f_{n^{\prime}}, g_{n^{\prime}}\right)\right| \leqslant$ $\left|\left(A f_{n^{\prime}}, f_{n^{\prime}}\right)\right|+\left|\left(A f_{n^{\prime}}, f_{n^{\prime}} \pm g_{n^{\prime}}\right)\right|$, we have $\left|\left(A f_{n^{\prime}}, g_{n^{\prime}}\right)\right| \leqslant\left|\left(A f_{n^{\prime}}, f_{n^{\prime}}\right)\right|+\varepsilon / 2$. But $\left(A f_{n^{\prime}}, f_{n^{\prime}}\right) \rightarrow 0$, and so $\left|\left(A f_{n^{\prime}}, g_{n^{\prime}}\right)\right|$ can be made less than $\varepsilon$ by choosing $n^{\prime}$ sufficiently large. Note that the case of finite $\left\{n^{\prime}\right\}$ is not of any interest. To the other part the considerations of case $\mathrm{I}$ apply, and thus the linearity of $N_{0}(A)$ is established.

Next, we prove the converse of the above result, that is, $N_{z}(T)$ is not a subspace of $l^{\infty}(H)$ if $z$ is not an extreme point of $W(T)^{-}$. If $z$ is an interior point of $W(T)^{-}$, then an easy application of the Embry's theorem that $M_{z}(T)$ is not linear if $z$ is not an extreme point of $W(T)$, shows that $N_{z}(T)$ is not linear. If $z$ is a nonextreme boundary point of $W(T)^{-}$, we carry out the reduction $A=\exp (i \theta) T$ $-z I$ as before. Hence there exist two sequences of unit vectors $\left\{f_{n}\right\}$ and $\left\{g_{n}\right\}$ such that $\left(A f_{n}, f_{n}\right) \rightarrow i a$ and $\left(A g_{n}, g_{n}\right) \rightarrow-i a$. We will now prove the existence of two distinct scalars $z_{1}$ and $z_{2}$ such that $\left\{f_{n}+z_{1} g_{n}\right\},\left\{f_{n}+z_{2} g_{n}\right\} \in N_{0}(A)$, to render the nonlinearity of $N_{0}(A)$ obvious. As in the first part of the proof, $h_{n}=A f_{n}+A^{*} f_{n} \rightarrow 0$. So $\left(A f_{n}+z A g_{n}, f_{n}+z g_{n}\right)=\left(A f_{n}, f_{n}\right)+|z|^{2}\left(A g_{n}, g_{n}\right)+$ $z\left(g_{n}, h_{n}\right)+2 i \operatorname{Im}\left(\bar{z}\left(A f_{n}, g_{n}\right)\right)$. All the terms on the right hand side converge except possibly $\operatorname{Im}\left(\bar{z}\left(A f_{n}, g_{n}\right)\right)$. But since $\left(A f_{n}, g_{n}\right)$ is bounded we can always choose a convergent subsequence. Assume that done for simplicity. So $\left(A f_{n}, g_{n}\right)$ $\rightarrow b+i c$, say. Hence $\left(A f_{n}+z A g_{n}, f_{n}+z g_{n}\right) \rightarrow i a\left(1-|z|^{2}\right)+2 i(c x-b y)$. So all points on the real circle $x^{2}+y^{2}+(2 / a)(b y-c x)-1=0$ will answer our purpose. This completes the proof of Theorem 1 .

\section{An example}

It is interesting to note that though $N_{z}(T)$ is linear if $z$ is an extreme point of $W(T)^{-}$, the following set, quite similar to $N_{z}(T)$, defined by

$$
N_{z}^{\prime}(T)=\left\{\left\{f_{n}\right\} \in l^{\infty}(H):\left(A f_{n}, f_{n}\right) /\left(f_{n}, f_{n}\right) \rightarrow z,\left\|f_{n}\right\| \neq 0\right\},
$$


does not, in general, have the property that $\left\{f_{n}+g_{n}\right\} \in N_{z}^{\prime}(T)$ if $\left\{f_{n}\right\},\left\{g_{n}\right\} \in$ $N_{z}^{\prime}(T)$. We give a counterexample.

Let $\left\{e_{n}\right\}$ and $\left\{e_{n}^{\prime}\right\}$ be disjoint sets of orthonormal elements of $H$. Define a linear operator $V$ such that $V e_{n}=e_{n}$ and $V e_{n}^{\prime}=(1 / n) e_{n}^{\prime}$. It is easy to verify that $V$ is selfadjoint.

Let $f_{n}=\left(e_{n}+n e_{n}^{\prime}\right) /\left(1+n^{2}\right)^{1 / 2}$, and $g_{n}=\left(e_{n}-e_{n}^{\prime}\right) /\left(1+n^{2}\right)^{1 / 2}$. Clearly $\left\|f_{n}\right\|=\left\|g_{n}\right\|=1$. Hence $V f_{n}=\left(e_{n}+e_{n}^{\prime}\right) /\left(1+n^{2}\right)^{1 / 2} \rightarrow 0$. Likewise, $V g_{n} \rightarrow 0$. Define $U=V^{2}$. So $\left(U f_{n}, f_{n}\right) /\left(f_{n}, f_{n}\right)$ and $\left(U g_{n}, g_{n}\right) /\left(g_{n}, g_{n}\right)$ tend to zero. But $\left(U\left(f_{n}+g_{n}\right),\left(f_{n}+g_{n}\right)\right) /\left\|f_{n}+g_{n}\right\|^{2}=\left\|V\left(f_{n}+g_{n}\right)\right\|^{2} /\left\|f_{n}+g_{n}\right\|^{2}=1$ for all $n$.

This proves our assertion.

\section{Weak convergence on $\partial W(T)$}

THEOREM 2. Let $\mu \in L \cap W(T)^{-}$, where $L$ is a line of support of $W(T)^{-}$at $\mu$ and $f_{n} \rightarrow f$ be a weakly convergent sequence such that $\left(T f_{n}, f_{n}\right) \rightarrow \mu$. Then either $f=0$ or $(T f, f) /(f, f) \in L \cap W(T)$.

Proof. Let $\xi$ be a point on the outward drawn normal at $\mu$. Define $A=T-\xi I$. Clearly, $|\mu-\xi|=\operatorname{Inf}|(A g, g)| /(g, g), g \in H$. Consider the operator $B=\bar{\lambda} A$ $+\lambda A^{*}-2|\lambda|^{2} I$, where $\lambda=\mu-\xi . B$ is easily seen to be positive and $\left(B f_{n}, f_{n}\right) \rightarrow$ 0 . Hence, by a property of positive operators $B f_{n} \rightarrow 0$. But $B f_{n} \rightarrow B f$ and so $B f=0$, by the uniqueness of weak limit. Therefore, $(B f, f)=2 \operatorname{Re}(\bar{\lambda}(A f, f))-$ $2|\lambda|^{2}(f, f)=0$. If $f \neq 0$, we have consequently that $\cos \theta|(A f, f) /(f, f)|$ $=|\lambda|$, where $\theta$ is the amplitude of $\bar{\lambda}(A f, f)$. So, in terms of $T$, we can assert that $(T f, f) /(f, f) \in L W(T)$.

The following corollary is a mild generalization of a result of Das [1977] and Garske [1979] in that is also holds for some extreme points of $W(T)$ which are not necessarily extreme points of $W(T)^{-}$.

COROLlaRY 3. Let $\lambda$ be a boundary point but not necessarily an extreme point of $W(T)^{-}$and let $L$ be a line of support of $W(T)$ at $\lambda$. If $L \cap W(T)=\{\lambda\}$, and $f_{n}-f$ is a weakly convergent sequence such that $\left(T f_{n}, f_{n}\right) \rightarrow \mu, \mu \in L \cap W(T)^{-}$, then either $f=0$ or $(T f, f) /(f, f)=\lambda$.

Proof. Follows immediately from Theorem 2 as $L \cap W(T)$ consists of a single point.

Let $\mu$ be an extreme point of $W(T)^{-}$and $f_{n}-f,\left\|f_{n}\right\|=1$ be such that $\left(T f_{n}, f_{n}\right) \rightarrow \mu$. Das [1977] and Garske [1979] have shown that either $f=0$ or 
$(T f, f) /(f, f)=\mu$. The situation may, however, be quite different if $\mu$ is a nonextreme boundary point of $W(T)^{-}$. It may very well happen that $\left(T f_{n}, f_{n}\right) \rightarrow \mu$ while $(T f, f) /(f, f)=\lambda \neq \mu$. An example of this may be found in Garske [1979]. While Theorem 2 and Corollary 3 above throw some light on the position $(T f, f) /(f, f)$, the following theorem (Theorem 4) gives a bound for the norm of the weak limit $f$ in such a case.

THEOREM 4. Let $f_{n} \rightarrow f$ be a weakly convergent sequence of unit vectors such that $\left(T f_{n}, f_{n}\right) \rightarrow \mu$ where $\mu \in \partial W(T)$. Then either

(i) $f=0$, or

(ii) $(T f, f) /(f, f)=\mu$, or

(iii) $\mu$ is not an extreme point of $W(T)^{-}$and $(f, f) \leqslant a / b$, where $a$ and $b$ are the distances from $\mu$ and $(T f, f) /(f, f)$ respectively to the extreme point of $W(T)^{-}$ collinear with $\mu$ and $(T f, f)$ and on the opposite side of $\mu$ from $(T f, f) /(f, f)$.

Proof. In light of the facts mentioned above, we need only discuss the case when $\mu$ is not an extreme point of $W(T)^{-}$and $(T f, f) /(f, f)=\lambda \neq \mu$. Since we may always carry out the transformation from $T$ to $A$ as in Theorem 2, we may assume without loss of generality that $0 \notin W(T)^{-}$and $|\mu|=\operatorname{Inf}|(T g, g)| /(g, g)$, $g \in H$. For any real $t$, we have

$$
\begin{gathered}
\left(T f+t T f_{n}, f+t f_{n}\right) /\left(f+t f_{n}, f+t f_{n}\right) \\
\rightarrow\left(\mu t^{2}+(2 t+1)(T f, f)\right) /\left(t^{2}+(2 t+1)(f, f)\right) \\
=\mu+(\lambda-\mu)(2 t-1)(f, f) /\left(t^{2}+(2 t+1)(f, f)\right) .
\end{gathered}
$$

If $\|f\|=1$, then $f_{n} \rightarrow f$, and as a result $\lambda=\mu$. So we may assume that $(f, f)<1$. Let $u=(2 t+1)(f, f) /\left(t^{2}+(2 t+1)(f, f)\right)$. It is easily verified that $u$ lies between 1 and $(f, f) /((f, f)-1)$. By Theorem $2, \lambda, \mu$ and $\mu+(\lambda-$ $\mu)(f, f) /((f, f)-1)$ lie on the same line segment. Clearly $\lambda$ and $\mu+(\lambda-$ $\mu)(f, f) /((f, f)-1)$ lie on opposite sides of $\mu$. Hence $|\lambda-\mu|(f, f) /(1-$ $(f, f)) \leqslant a$, or $(f, f) \leqslant a /(|\lambda-\mu|+a)=a / b$. This brings the proof to an end.

\section{Acknowledgments}

The authors are grateful to Dr. E. N. Dancer, Dr. J. J. Koliha and Dr. B. Sims for fruitful discussions. They also gratefully appreciate the suggestions of the referee which helped to reduce the proof of Theorem 1 besides improving the overall presentation of the paper. 


\section{References}

K. C. Das (1977), 'Boundary of numerical range,' J. Math. Anal. Appl. 60, 779-780, MR 56 \#6431.

M. R. Embry (1970), 'The numerical range of an operator,' Pacific J. Math. 32, 647-650, MR 41 $\# 4260$.

M. R. Embry (1975), 'Orthogonality and the numerical range,' J. Math. Soc. Japan 27, 405-411, MR $52 \# 1352$.

G. Garske (1979), 'The boundary of the numerical range of an operator,' J. Math. Anal. Appl. 68, 605-607.

J. G. Stampfli (1966), 'Extreme points of the numerical range of a hyponormal operator,' Michigan Math. J. 13, 87-89, MR 32 \#551.

Department of Mathematics

University of Melbourne

Parkville, Victoria 3051

Australia
Present address of K. C. Das:

Department of Mathematics

Indian Institute of Technology

Kharagpur 721302, W. Bengal

India 